\title{
Combining global positioning system and accelerometer data to determine the locations of physical activity in children
}

\author{
Nicolas M. Oreskovic ${ }^{1,2,3}$, Jeff Blossom ${ }^{4}$, Alison E. Field ${ }^{3,5}$, Sylvia R. Chiang ${ }^{2}$, Jonathan P. \\ Winickoff ${ }^{1,2,3}$, Ronald E. Kleinman ${ }^{2,3}$ \\ ${ }^{1}$ MGH Center for Child and Adolescent Health Research and Policy, Boston, MA USA; ${ }^{2}$ Massachusetts General \\ Hospital, Boston, MA, USA; ${ }^{3}$ Harvard Medical School, Boston, MA, USA; ${ }^{4}$ Harvard Center for Geographic \\ Analysis, Cambridge, MA, USA; ${ }^{5}$ Children's Hospital Boston, Boston, MA, USA
}

\begin{abstract}
National trends indicate that children and adolescents are not achieving sufficient levels of physical activity. Combining global positioning system (GPS) technology with accelerometers has the potential to provide an objective determination in locations where youth engage in physical activity. The aim of this study was to identify the optimal methods for collecting combined accelerometer and GPS data in youth, to best locate where children spend time and are physically active. A convenience sample of 24 mid-school children in Massachusetts was included. Accelerometers and GPS units were used to quantify and locate childhood physical activity over 5 weekdays and 2 weekend days. Accelerometer and GPS data were joined by time and mapped with a geographical information system (GIS) using ArcGIS software. Data were collected in winter, spring, summer in 2009-2010, collecting a total of 26,406 matched datapoints overall. Matched data yield was low (19.1\% total), regardless of season (winter, $12.8 \%$; spring, $30.1 \%$; summer, $14.3 \%$ ). Teacher-provided, pre-charged equipment yielded the most matched (30.1\%; range: $10.1-52.3 \%)$ and greatest average days (6.1 days) of data. Across all seasons, children spent most of their time at home. Outdoor use patterns appeared to vary by season, with street use increasing in spring, and park and playground use increasing in summer. Children spent equal amounts of physical activity time at home and walking in the streets. Overall, the various methods for combining GPS and accelerometer data provided similarly low amounts of combined data. No combined GPS and accelerometer data collection method proved superior in every data return category, but use of GIS to map joined accelerometer and GPS data can demarcate childhood physical activity locations.
\end{abstract}

Keywords: physical activity, built environment, adolescence, global positioning system, geographical information system, USA.

\section{Introduction}

The prevalence of childhood obesity has reached epidemic proportions and ranks among the most prevalent chronic conditions of children (Perrin et al., 2007). In the United States of America (USA), over the past several decades, the rate of obesity has increased to $17 \%$ among children aged 12 to 19 years (Ogden et al., 2010). The relatively rapid weight increase in the population suggests environmental causes exacerbating a genetic predisposition (Barlow and Expert Committee, 2007; Krebs et al., 2007).

National trends indicate insufficient and declining levels of physical activity among children in the USA,

Corresponding author:

Nicolas M. Oreskovic

MGH Center for Child and Adolescent Health Policy

100 Cambridge Street, 15th Floor

Boston, Massachusetts 02114, USA

Tel. +1 617726 0593; Fax + 16177261886

E-mail: noreskovic@partners.org with older children being the least active (Nader et al., 2008). Active use of the built environment, such as walking to school or to a friend's house, using outdoor fields or playgrounds for play, etc. may produce a small daily increase in physical activity (Cohen et al., 2006; Epstein et al., 2006). The American Academy of Pediatrics recently released a policy statement highlighting the important interplay of the built environment and children's opportunities for physical activity (Committee on Environmental Health, 2009).

Many studies have described associations between built environment, physical activity and weight status. Several built environment features, which lend themselves well to geographic analysis, have consistently been shown to be associated with physical activity and the degree of obesity in children. Such features include parks, playgrounds, school spaces, green spaces as well as attributes such as distance to school, presence of sidewalks, street connectivity and "walkability" in general (Cooper et al., 2005; Frank et al., 2005; Cradock et al., 2007; Papas et al., 2007; Lee et al., 2008; Oreskovic et al., 2009) These built environment 
features are thought to increase physical activity through promotion of physical activity, walking and possibly riding a bicycle within a given space.

Studies assessing associations between physical activity in children and built environment have used self-report, direct observation, or geographical information system (GIS) to look for correlations between an individual's surrounding built environment and physical activity levels. Limitations to using these methods include subjective data and recall bias with self-report, inability to assess an individual's total daily physical activity with direct observation, and possible ecological fallacy with GIS studies, e.g. the potential to incorrectly assign physical activity to a particular built environment variable being tested in cross-sectional analyses. Little is known about the types of built environments in which children are active, and the amount of time and physical activity they expend in these spaces.

Recent studies have begun using global positioning system (GPS) receivers combined with accelerometers to better understand children's physical activity and movement patterns. Early work in this field indicates that GPS mapping technology combined with accelerometers can differentiate among different types and locations of physical activity among children (Jones et al., 2009; Cooper et al., 2010; Quigg et al., 2010). Data collection remains difficult with these new modalities, especially among children, in part due to the short battery life of the GPS receivers. Previous studies have tried different methods for data collection with varying success, and there is no emerging consensus regarding a "gold standard" in this field. However, the use of accelerometer and GPS together could not only reveal the degree at which children are physically active, but also show the locations in which they are the most and the least active. This approach would allow objective assessment of which built environment features most influence child movement patterns, thus providing information that could ultimately be used to design neighbourhoods that encourage physical activity. Identifying feasible and optimal data collection methods are initial steps promoting studies capable of obtaining a more complete picture of childhood physical activity.

We describe the combined use of GPS and accelerometers to assess the locations of mid-level school children throughout the day, including the degree of physical activity spent in each location. The aim of this study was to test the feasibility of collecting and analysing combined GPS and accelerometer data by comparing several different approaches to data collection and to determine which methodology provides the richest data.

\section{Materials and methods}

\section{Participants}

Mid-level school children aged 11 to 12 years living in Revere, Massachusetts were recruited for this study taking place over three data collection phases; winter (December 2009), spring (March 2010) and summer (July 2010). Participation was voluntary, and parental written informed consent was obtained along with child assent. The study was approved by the Partners Health Care Institutional Review Board.

\section{Location}

The city of Revere is a densely populated (3,512 persons $/ \mathrm{km}^{2}$ ), residential community located in Suffolk county, Massachusetts about $8 \mathrm{~km}$ northeast of Boston, with the Atlantic Ocean to the East. It has a population of 47,000 consisting of $47 \%$ Caucasians, 37\% Hispanic Americans, 4\% African Americans, 7\% Asian Americans and a 4\% multi-racial part, including a sizeable immigrant population. The median, annual household income is US\$ 49,178. The city comprises $25.88 \mathrm{~km}^{2}$, of which 900 acres (about 3.63 $\mathrm{km}^{2}$ ) are open water or wetlands and 4,054 acres (about $16.32 \mathrm{~km}^{2}$ ) are developed land, $70 \%$ of which is used for housing. Revere beach was the first public beach in the USA, and the city has three subway stations on the metropolitan Boston public transportation system. Average temperatures are $28.6^{\circ} \mathrm{F}\left(-2^{\circ} \mathrm{C}\right)$ in January and $73.5^{\circ} \mathrm{F}\left(+23^{\circ} \mathrm{C}\right)$ in July, with an annual precipitation of $1,054 \mathrm{~mm}$.

\section{Instruments and measures}

Physical activity data were measured with an Actigraph GT1M accelerometer (http://www.theactigraph.com/) worn around the hip, set at 30-sec epochs and collapsed into 1-min intervals for analysis. Location data was recorded with a Forerunner 201 GPS mapping device (http://www8.garmin.com/products/forerunner201/) worn around the wrist. The Forerunner 201 records location data when direction or speed is changed resulting in a point being recorded every 3 to $55 \mathrm{sec}$. One-minute intervals of GPS data were created, with the first GPS datapoint for every minute used for analysis. Children were instructed to wear both monitors for 5 weekdays and 2 weekend 
days during all hours they were awake except during contact sports and water activities. They were also asked to keep an activity log throughout the week.

\section{Built environment features}

We chose to classify the mapped GPS locations into six built environment categories given either as "home", "school", "car", "indoor/other" (non-home, non-school), "park/playground" (which included all outdoor recreation spaces) or "street/walking". We chose these geographic feature categories based on prior studies demonstrating their associations with physical activity and weight status in children (Papas et al., 2007) as well as the ability to clearly distinguish between these different features using GIS software. Home and school locations were determined by address geocoding, resulting in discrete points representing each subject's home and school on the map. For classification of GPS locations such as the indoors, park or playground, and street classification categories, photo interpretation from high resolution (30 $\mathrm{cm}$ pixel) color aerial orthophotos (flown in April of 2008) were used. Buildings, parks, playgrounds and streets are all easily identifiable on these aerial photos.

\section{Data collection}

We tested several different approaches to distributing and collecting the GPS and accelerometer devices during three separate waves of data collection. For each approach, staff received similar training in distributing and instructing children on how to wear the devices. All participating children received the same instructions and training on how to wear the devices. The investigation was carried out in three "waves":

Winter wave: a research assistant (RA) met participants at school each morning in winter. Accelerometers were distributed on the first day of the study and collected after the conclusion of the study. For the GPS, as the instrument had a battery life of up to 16 hours, the RA distributed a charged GPS and collected the depleted GPS devices each morning. Lost accelerometers and GPS instruments were replaced during the study.

Spring wave: a school teacher was recruited to distribute and collect study materials in spring. The accelerometers were distributed on the first day of the study and collected on the last day of the study. The teacher distributed charged GPS devices and collected the depleted ones during class time at the end of each school day. Lost accelerometers and GPS instruments were replaced during the study.

Summer wave: a camp counselor distributed accelerometers and GPS devices to children on the first day of a summer programme, instructed them on how to charge the GPS instruments at home overnight and collected both devices at the conclusion of the study. The counselor verbally reminded children no to forget to charge the instruments overnight at the end of each camp day. Lost accelerometers and GPS were replaced during the study.

Through an exit survey, the children were asked to report on their level of ease and satisfaction with wearing the accelerometer and GPS devices, as well as their level of satisfaction overall with their participation in the study. Parents did not receive any training or instructions.

\section{Data processing and analysis}

Data on physical activity and built environment were cross-sectional. The output from each child's accelerometer containing physical activity data was matched and joined to the child's GPS output containing location data using the date field from both datasets in day/hour/min format. To allow for timematching, the start time of each GPS datapoint was rounded down to the nearest min to match the start time of each accelerometer epoch. This matching process involved the following approach:

(i) for each accelerometer used, information regarding "activity count", "horizontal activity", and "step count" as well as the date and time, were exported into "comma separated value" (CSV)formatted files by means of ActiLife software; and

(ii) for each GPS, the DNR Garmin software was used to export information about the longitude, latitude, date and time into CSV-formatted files:

a) a geodatabase file was created using ArcGIS version 9.2 software (ESRI, Redlands, CA, USA), importing the accelerometer CSV files as tables and the date and time fields as "string" type. The GPS CSV files were imported into the geodatabase as point feature classes, using the "add XY data" command to specify point locations corresponding to the longitude and latitude coordinates. The GPS date and time fields were imported as "string" type;

b) using text parsing functions available within ArcGIS, the accelerometer date and time fields and GPS date and time fields were converted into the same format, and merged into one field: Date_Time; and 
c) for each subject's data, the accelerometer table was joined with the GPS feature class using the respective Date_Time fields. This produced locations for accelerometer "activity count", "horizontal activity", and "step count" values where the accelerometer Date_Time value matched a GPS Date_Time value.

The accelerometer data provided the maximum possible number of datapoints, while the GPS datapoints determined the ultimate number of combined accelerometer-GPS datapoints. Only the matched data were retained and analysed. We calculated the percent of matched data for each subject. To best capture the children's activities and use the maximum amount of matched data available, we required that they provided at least one full hour of matched data recorded on at least one of the seven measurement days to be included in the analysis.

The matched GPS and accelerometer data were combined into a single dataset and imported into ArcGIS9.2 as a file geodatabase. Each child's home address was geocoded using Google Maps, accessed through the website (http://batchgeo.com/) in December 2010. Each child's locations throughout the day were displayed within ArcGIS by a GIS specialist who coded the locations as either "home", "school", "car", "indoor/other" (non-home, non-school), "park/play- ground" (which included all outdoor recreation spaces) or "street/walking" (Fig.1 and 2). This location classification procedure involved downloading the Commonwealth of Massachusetts' Office of Geographic Information (MassGIS) $30 \mathrm{~cm}$ pixel resolution, color, digital orthoimagery for Revere (http://www.mass.gov/mgis/colororthos2008.htm), and loading this imagery into the ArcMap module of ArcGIS. This highly detailed color imagery was captured in 2008 at an accuracy of $3 \mathrm{~m}$. Each subject's GPS data, home location and school location were overlaid on the imagery into a custom ArcMap map document. For each subject, classifying the GPS locations preceded in a hierarchical fashion as described below. First, the subject's home location was navigated to on the map. All GPS points within $25 \mathrm{~m}$ of the center of the house were selected and classified as home. Often this $25 \mathrm{~m}$ radial distance had to be increased to include larger GPS location distributions around the home. Next the school location was navigated to classifying all GPS points within $100 \mathrm{~m}$ of the school perimeter as school. The remaining locations were classified through photo interpretation. Areas containing GPS points were visually scanned at the 1:4,000 scale and based on photo interpretation of the underlying imagery, interpreting the locations as either "indoor/other", "park/playground" or "street/walk-

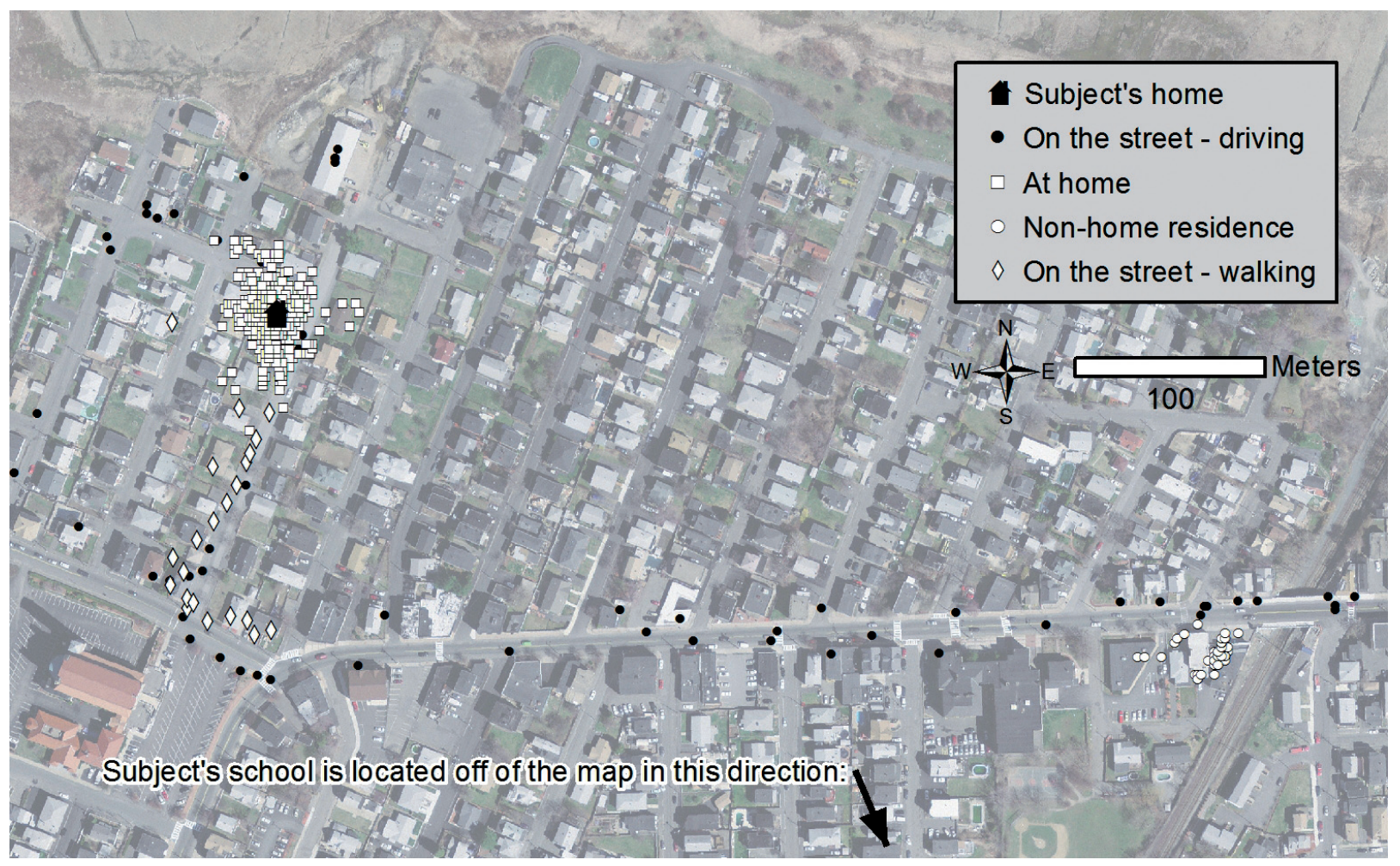

Fig. 1. GIS mapping of combined GPS and accelerometer data. 

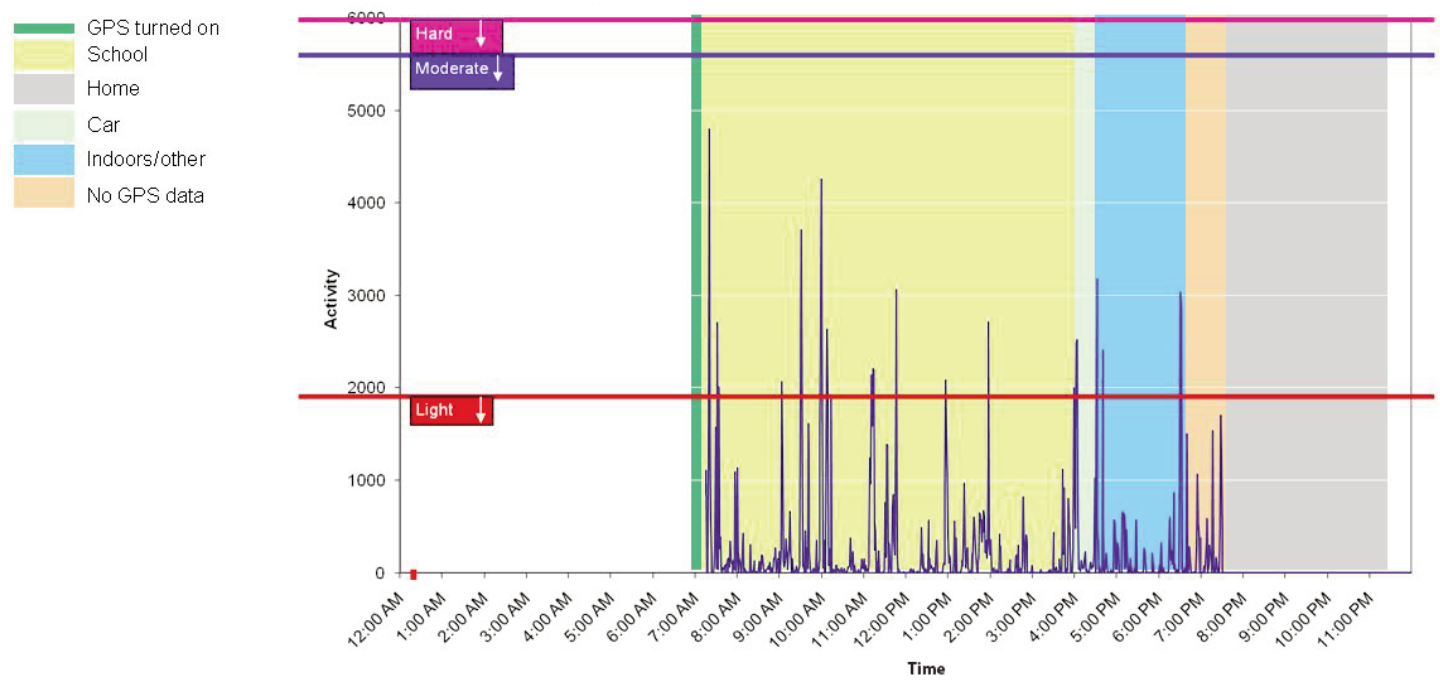

Fig. 2. GPS classification of accelerometer data.

ing”. Ideally, using a point in polygon spatial overlay function to automatically determine which class each GPS point fell into would be a more efficient, automated classification method. However, this was not possible due to the lack of mapped "indoor/other" and "park/playground" vector layers; instead visual scanning and photo interpretation from the aerial photos was chosen as the best option. The street locations were further classified into "car" or "street/walking". This involved inspecting the distance spacing on the map between chronologically ordered GPS point locations falling in or near a street. GPS points greater than roughly $70 \mathrm{~m}$ apart were classified as "car"; points less apart than that were classified as "street/walking". This criterion was used due to the GPS points being recorded at one minute intervals, and $70 \mathrm{~m}$ per minute is a proximal estimate of average walking speed $(5 \mathrm{~km} / \mathrm{hr})$.

Each location-coded accelerometer datapoint was classified into one of four intensity categories: "0 counts/min" (no activity), "1-1951" (light activity), "1952-5724" (moderate activity) and " $\geq 5,725$ " (vigorous activity). These count/min cutoff points are based on the age-specific equation of Freedson et al. (1998, 2005), and relate broadly to moderate physical activity defined as $\geq 3$ metabolic equivalents of task (METs) and vigorous activity defined as $\geq 6$ METs. Each child's total daily physical activity and daily minutes of moderate-to-vigorous physical activity (MVPA) were then calculated within each mapped location. Finally, descriptive statistics were used to calculate total time spent in each location, total combined time at all levels of physical activity (light, moderate and vigorous) in each location, and total time in moderate-to-vigorous physical activity in each location. In addition, the daily temperatures were recorded for each day that children participated in the study.

\section{Results}

\section{Participants and data collection}

A convenience sample of 26 mid-level school children in Revere, USA were invited to participate in the study, all of whom provided parental consent and were included in the study. The average daily temperature during data collection was $34^{\circ} \mathrm{F}$ with a daily range of $28-40^{\circ} \mathrm{F}\left(1.1^{\circ} \mathrm{C}\right.$, range: $\left.-2.2-4.4\right)$ during winter, $54^{\circ} \mathrm{F}$ with range $44-71\left(12.2^{\circ} \mathrm{C}\right.$, range: 6.6$21.6^{\circ} \mathrm{C}$ ) during spring and $77^{\circ} \mathrm{F}$ with range $68-88$ $\left(25.0^{\circ} \mathrm{C}\right.$, range $\left.20.0-31.1^{\circ} \mathrm{C}\right)$ in the summer.

Some minor problems connected with the data collection were noted:

Winter wave: one child lost an accelerometer. One child lost his GPS mapping device and did not return to collect a replacement GPS. Both subjects were thus excluded from analyses due to lack of matched data.

Spring wave: one child lost an accelerometer and did not collect a replacement and was thus excluded from analyses due to lack of matched data. One child lost one GPS mapping device, and four subjects returned GPS devices whose unique identification numbers were not possible to be determined, making location data collected on those days unusable. 
Summer wave: One child lost one GPS mapping device and four subjects returned GPS devices without unique identification numbers making the location data collected on those days unusable.

In total, 24 children (aged 11-12 years, $41.7 \%$ male), eight per wave, provided matched GPS and accelerometer data on at least one measurement day and were included in the analyses. Children reported similar levels of satisfaction with the study across season (data not shown).

\section{GPS and accelerometer data matching}

A total of 26,406 matched datapoints were collected in the overall study, but with only $19.1 \%$ of the total available data successfully matched. This low match rate is believed to primarily be due to the four reasons below:

(i) inability to record GPS locations while indoors;

(ii) lengthy (several minutes in many cases) GPS signal re-acquisition time when transitioning from indoors to outdoors;

(iii) inability to specify the collection interval on the GPS model used; and

(iv) GPS recording a location only when movement (positional change) was detected.

Winter wave: some 5,123 datapoints were matched with $12.8 \%$ (daily range $=0.5-54.7 \%$ ) of the available winter data successfully matched. On average, participants returned 4.9 days of matched data.

Spring wave: some 19,607 datapoints were matched with $30.1 \%$ (daily range $=10.1-52.3 \%$ ) of the available data matched. Participants returned an average of 6.1 days of matched data.

Summer wave: some 1,676 datapoints were matched with $14.3 \%$ (daily range $=0.4-60.4 \%$ ) of the data being matched. On average, participants returned 2.5 days of matched data.

\section{Location patterns and physical activity locations}

We successfully assigned a built environment location code for all of the combined data based on geocoding and photo interpretation within a GIS. Overall, children spent most of their time at home, and this remained constant across the seasons (Table 1). Stratification of the locations data by season, however, illustrated that children did tend to use different built environments during different seasons. During winter, the children spent the overwhelming majority of their time at home. This was generally also true for spring and summer though they also spent a notice- able amount of time in other indoor sites and in the streets in the spring and a lot of time at parks and playgrounds in the summer.

When looking only at time spent in physical activity, the children spent similar amounts of time at home and in the streets (Table 2). In wintertime, the physical activity was most often recorded as taking place at home $(52 \%)$, but time was also school $(15 \%)$, while in summer they were most often physically active in parks and playgrounds (45\%).

Overall, judging from the matched data, the children were most likely to be engaged in moderate-tovigorous physical activity when they were outdoors in the streets (Table 2). In winter, most of the MVPA was recorded at home, while in spring most occurred outdoors in the streets. During summer, most MVPA was recorded in parks and playgrounds. On average for the whole period of investigation, $15.4 \mathrm{~min}$ a day (range 0-133 min) of MVPA was recorded. However, differences in MPVA levels by season were found with children in the spring wave group showing the greatest number of minutes of MPVA per day, i.e. $36.4 \mathrm{~min}$ $\mathrm{MVPA} /$ day (range $=7.9-133 \mathrm{~min}$ ) as opposed to winter (1.7 $\mathrm{min}$ of MVPA/day with a range of $0-5.2 \mathrm{~min}$ ) and summer (6.3 min of MVPA/day with a range of 0 $12.7 \mathrm{~min}$ ). When we categorized the average daily minutes of MVPA by location, parks and playgrounds appeared to be the location where the children spent the highest number of minutes of MVPA/day on average, albeit with a wide spread of measurements, each category containing multiple outliers (Fig. 3). Given the relatively small amount of data points available for average minutes of MVPA/day by location and the presence of numerous outliers, we did not evaluate the seasonal variations.

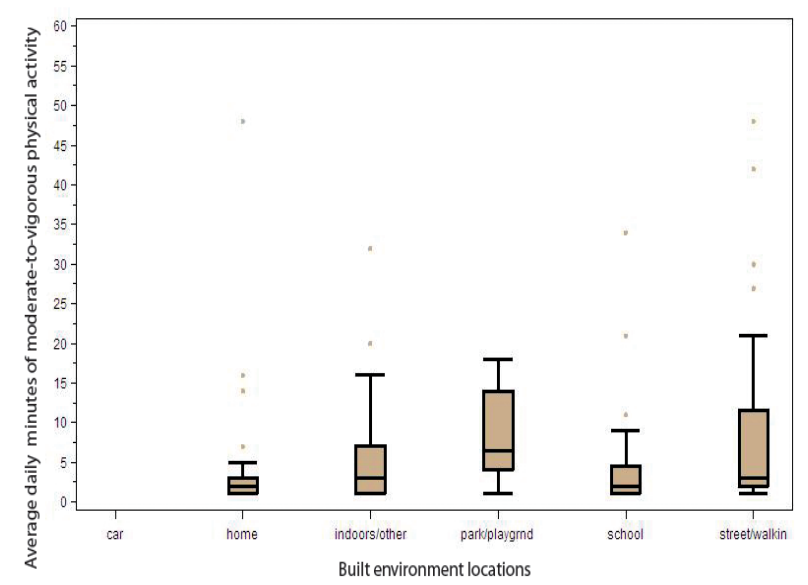

Fig. 3. Level of moderate-to-vigorous physical activity (MVPA), stratified by subject location. 
Table 1. Locations of matched GPS and accelerometer data, overall and by season.

\begin{tabular}{lrrrr}
\hline \multirow{2}{*}{ Location } & \multicolumn{1}{c}{ Total } & \multicolumn{1}{c}{ Winter } & \multicolumn{1}{c}{ Spring } & Summer \\
\cline { 2 - 4 } & Minutes (\%Time) & Minutes (\%Time) & Minutes (\%Time) & Minutes (\%Time) \\
\hline Car & $2,123(8.0)$ & $80(1.6)$ & $1,825(9.3)$ & $218(13.0)$ \\
Home & $12,929(49.0)$ & $3,877(75.7)$ & $8,352(42.6)$ & $700(41.8)$ \\
Indoors/other & $4,186(15.8)$ & $476(9.1)$ & $3,643(18.5)$ & $76(4.5)$ \\
School & $1,570(6.0)$ & $170(3.3)$ & $1,400(7.1)$ & - \\
Park/playground & $1,520(5.8)$ & $64(1.3)$ & $920(4.7)$ & $536(32.0)$ \\
Street/walking & $4,078(15.4)$ & $465(9.0)$ & $3,467(17.6)$ & $146(8.7)$ \\
\hline
\end{tabular}

Table 2. Total amount and percent of children's physical activity by location, season, and physical activity level.

\begin{tabular}{|c|c|c|c|c|}
\hline \multirow{2}{*}{ Location } & Total & Winter & Spring & Summer \\
\hline & Minutes (\% Time) & Minutes (\% Time) & Minutes (\% Time) & Minutes (\%Time) \\
\hline \multicolumn{5}{|c|}{ All physical activity levels location* } \\
\hline Car & $656(8.7)$ & $30(2.2)$ & $595(10.9)$ & $31(4.2)$ \\
\hline Home & $2,298(30.4)$ & 696 (51.6) & $1360(24.9)$ & $242(32.6)$ \\
\hline Indoors/other & $756(10.0)$ & $210(15.6)$ & $498(9.1)$ & $48(6.5)$ \\
\hline School & $887(11.8)$ & $83(6.2)$ & 804 (14.7) & - \\
\hline Park/playground & $631(8.4)$ & $1(0.1)$ & $300(5.5)$ & $330(44.5)$ \\
\hline Street/walking & $2,324(30.8)$ & $330(24.4)$ & $1903(34.9)$ & $91(12.3)$ \\
\hline \multicolumn{5}{|c|}{ Moderate-to-vigorous physical activity** } \\
\hline Car & - & - & - & - \\
\hline Home & $670(33.5)$ & $28(43.1)$ & $619(33.8)$ & $23(12.3)$ \\
\hline Indoors/other & $114(5.7)$ & $10(15.4)$ & $93(5.1)$ & $11(10.2)$ \\
\hline School & $169(8.4)$ & $8(12.3)$ & $161(8.8)$ & - \\
\hline Park/playground & $217(10.8)$ & - & $155(8.5)$ & $62(57.4)$ \\
\hline Street/walking & 833 (41.6) & $19(29.2)$ & $802(43.8)$ & $12(11.1)$ \\
\hline
\end{tabular}

*'All Physical Activity Levels' includes any combined datapoint with a non-zero accelerometer count; **'Moderate-to-Vigorous Physical Activity' defined as any accelerometer count > 1952, per Freedson equation: Feedson et al. (2005).

\section{Discussion}

We tried several different approaches collecting combined GPS and accelerometer data to determine the optimal method to successfully obtain information on the locations used for physical activity by school children in Massachusetts. We found that the overall return level of data was low with some variation by season and collection method. However, we were unable to decide whether the observed variations were due to seasonal effects, individual participant variability or methodological differences, neither did any specific type of data collection wave prove superior with regard to equipment loss, number of days of data provided, total number of matched datapoints, percent matched data or participant satisfaction.

Our goal was to test different methods of collecting combined GPS and accelerometer data in mid-level school children, using this cutting-edge methodology to obtain objective information on children's locations and use of the built environment for physical activity. While prior studies have been successful in combining GPS and accelerometers to collect data on movement patterns in children (Jones et al., 2009; Cooper et al., 2010; Quigg et al., 2010), each study employed its own unique collection methods with a wide range of returned data between the studies. As there is so far no consensus on a common procedure in this nascent field, we tried three different data collection approaches to identify whether one particular approach would provide a higher amount of combined data than the others. Accordingly, we did not report physical activity levels from accelerometer data alone as such data are widely available (Nader et al., 2008; Troiano et al., 2008), but rather opted to report all data jointly; the novel collection methodology we sought to optimize (Freedson et al., 1998, 2005).

Overall, although no children were lost to follow-up 
and only two children were excluded from the analysis for failing to provide any data, multiple subjects did provide incomplete data from partially missing data on physical activity or location. We tested several different approaches to collecting combined physical activity and location data over three different waves. For the distribution and collection of equipment, we tried different staff (RA, teacher and counselor), different times of the day, different locations, different ways to recharge the instruments (study staff versus subjects), and different ways reminding the subjects to wear the devices (no reminder in first wave, being in class with the teacher running the study in second wave, having a camp counselor provide daily reminders in the third wave). In general, we found that children who had their GPS instruments charged for them, provided more complete data than those who recharged the devices on their own. Participants who received instruments from, and returned instruments to, their school teacher provided the greatest amount of data, both in terms of total matched datapoints and the average number of days of data provision. Interestingly, participants in summer provided the least amount of matched datapoints and the fewest days of data provision, indicating that cold weather may not be the main factor deterring data collection and protocol adherence.

With regard to location classification, we were able to successfully assign a built environment location to all of the children's joined datapoints, providing objective information on where children spend time and exert physical activity. The availability of the MassGIS imagery enabled us to assign precise location classifications through consistent, standardised image interpretation techniques performed by a single GIS specialist. Our preliminary findings suggest that, overall, children spent most of their time, and also recorded most of their physical activity at home, although very little of this activity reached the MVPA range. Our findings are somewhat in contrast to a study of children in South West England, which found children's outdoor physical activity to be greater than their recorded indoor activity (Cooper et al., 2010). Our findings that MVPA occurred more often outdoors than at home may partly explain these differences and, taken together, suggest that efforts to encourage childhood activity should focus on maximizing outdoor activity. The different findings may partly reflect distinct climates; our study was conducted in northeastern USA, where temperatures are frequently below freezing through much of winter and spring is often cold, making outdoor activity less enticing. The finding that children recorded more time at home in the summer, however, suggest that these differences may not solely be due to climate. However, since prior studies combining GPS and accelerometer in children were conducted in England and New Zealand (Jones et al., 2009; Cooper et al., 2010; Quigg et al., 2010), the differences observed may also reflect different societal attitudes towards space and physical activity.

Our preliminary findings suggest some seasonal differences in physical activity levels and locations of physical activity among mid-level school children. These findings are consistent with a study based on combined GPS and accelerometer data by Cooper et al. (2010), in which greater outdoor physical activity was observed in children during summer than in winter. Although testing for differences in physical activity levels was not the primary goal of our study, the data suggest that mid-level school children may use different built environments to achieve physical activity during different seasons. The identification of a common procedure for combing location and physical activity data would allow better categorization of physical activity by season. It would also determine if and how season and temperature affect children's use of the built environment. While mid-level school children in this study were noted to spend a large amount of time at home during the cold winter months, they were noted to spend more time outdoor on streets during spring as temperatures warmed. This pattern continued to change during the warm summer months as they were then found to spend most of their time outdoors using spaces purposefully designed to promote outdoor activities such as parks and playgrounds. These findings have several implications. First, if children and adolescents in fact do vary their indoor and outdoor activities depending on temperature and season, it may be useful to incorporate this information into efforts and interventions that aim to promote physical activity by maximizing indoor physical activity in colder months and outdoor activities in the warmer months. Second, given the low recorded levels of outdoor physical activity in winter, it would be useful to gain a better understanding of what makes outdoor spaces more desirable in the winter for activities such as sledding and skating. Finally, if children and adolescents exhibit different patterns for outdoor spaces during the different seasons of the year, for instance favouring the use of spaces that promote active transportation in the spring (e.g. streets with sidewalks) versus spaces that promote active play (e.g. playgrounds and parks) 
in the summer, it may be worthwhile to build a variety of outdoor built environments that support the different profiles of outdoor activities, allowing children and adolescents to remain active outdoors throughout the year.

\section{Limitations}

Although available on newer instruments, time intervals could not be set on the Forerunner 201 GPS receiver used, which would have permitted us to better match accelerometer epochs to the GPS recordings resulting in a more complete matched dataset with fewer missing datapoints. Since the GPS receiver has a poor location capture rate indoors and most indoor activity had to be purposefully classified as indoor activity, the missing, matched datapoints in our dataset may have produced a bias towards outdoor activity. However, we were often able to capture when a subject entered a building and when he/she exited, thus allowing us to infer that all the physical activity occurring between these two datapoints occurred within the indoor location, allowing us to ascribe a location to the missing data. It is not ideal to use location classification based on imagery captured in 2008 for data collected in 2010, as the built environment could have changed during this interval. Additionally, photo interpretation is labour intensive. It might have been more efficient to use an automated GIS polygon overlay location classification technique. However, the primary aim of this study was to describe three different methodological approaches to combing GPS and accelerometer data and all qualitative data, despite showing possible differences by season and location, are secondary findings. Different children participated in each of the three waves of data collection, further limiting our ability to draw any robust conclusions about seasonal variations in use of the built environment for physical activity. Although we were not able to directly ascribe the effect of season/weather on an individual's variations in physical activity levels or locations, our data suggest the presence of seasonal variations. Further studies should explore how children's physical activity levels and locations change with the weather.

Age may be another limitation to collecting combined GPS and accelerometer data, which requires that participants follow fairly strict study protocol, including remembering wearing the devices daily, not losing the devices or handling them carelessly. There is an age limit, below which children cannot responsibly participate in data collection due to the risk for equipment loss or damage. We opted not to involve parents in this study and focused exclusively on children's ability to wear equipment and provide data. Providing parents with instructions on how children should wear the equipment may ensure greater study compliance and improve data return.

Finally, although the data seem to suggest that activity patterns and locations vary by season, this may be due to the fact that the data returned varied by season, at least partly. For instance, children in the summer wave only provided two and a half day's worth of data on average, compared to six days in the spring. This denominator is important since we report total physical activity levels and total MVPA levels by location. It may appear that children spend less total time in physical activity and less time being active in certain locations during the summer, but our results may simply represent a data collection bias. Further studies that test different data collection methods, including different recruitment sites (i.e. schools versus doctor's office), during different seasons, in different geographic settings, and among different populations should help finding an optimal common procedure for collecting maximum information on the objective locations of physical activity among youth.

\section{Conclusion}

Using several different data collection techniques, we found no clearly superior method for collecting combined accelerometer and GPS data. There may be seasonal variations in how children use different indoor and outdoor spaces. The children investigated spent most of their time at home with none or only light physical activity. Given the declining levels of physical activity and increasing rates of obesity among children in the USA (and in other places), a reliable common procedure to objectively track childhood movement patterns is urgently needed. This would facilitate the understanding of how and why children choose to use certain indoor and outdoor environments for physical activity, and how these patterns vary throughout the year.

\section{Acknowledgements}

Supported in part by the Department of Pediatrics, Massachusetts General Hospital. We thank the Revere Food and Fitness collaboration and Revere CARES for their input and guidance throughout the study, as well as Daniel Rodriguez, $\mathrm{PhD}$, for his kind guidance and assistance in conceptualizing and developing this study. 


\section{References}

Barlow SE and Expert Committee, 2007. Expert committee recommendations regarding the prevention, assessment, and treatment of child and adolescent overweight and obesity: summary report. Pediatrics 120, 164-192.

Cohen DA, Ashwood JS, Scott MM, Overton A, Evenson KR, Staten LK, Porter D, McKenzie TL, Catellier D, 2006. Public parks and physical activity among adolescent girls. Pediatrics 118, e1381-1389.

Committee on Environmental Health, 2009. The built environment: designing communities to promote physical activity in children. Pediatrics 123, 1591-1598.

Cooper AR, Page AS, Wheeler BW, Hillsdon M, Griew P, Jago R, 2010. Patterns of GPS measured time outdoors after school and objective physical activity in English children: the PEACH project. Int J Behav Nutr Phys Act 7, 31.

Cooper AR, Andersen LB, Wedderkopp N, Page AS, Froberg K, 2005. Physical activity levels of children who walk, cycle, or are driven to school. Am J Prev Med 29, 179-184.

Cradock AL, Melly SJ, Allen JG, Morris JS, Gortmaker SL, 2007. Characteristics of school campuses and physical activity among youth. Am J Prev Med 33, 106-113.

Epstein LH, Raja S, Gold SS, Paluch RA, Pak Y, Roemmich JN, 2006. Reducing sedentary behavior: the relationship between park area and the physical activity of youth. Psychol Sci 17, 654-659.

Frank LD, Schmid TL, Sallis JF, Chapman J, Saelens BE, 2005. Linking objectively measured physical activity with objectively measured urban form: findings from SMARTRAQ. Am J Prev Med 28, S117-125.

Freedson P, Pober D, Janz KF, 2005. Calibration of accelerometer output for children. Med Sci Sports Exerc 37, S523-530.

Freedson PS, Melanson E, Sirard J, 1998. Calibration of the computer science and applications, Inc. Accelerometer. Med Sci Sports Exerc 30, 777-781.

Jones AP, Coombes EG, Griffin SJ, van Sluijs EM, 2009. Environmental supportiveness for physical activity in English schoolchildren: a study using global positioning systems. Int J Behav Nutr Phys Act 6, 42.

Krebs NF, Himes JH, Jacobson D, Nicklas TA, Guilday P, Styne D, 2007. Assessment of child and adolescent overweight and obesity. Pediatrics 120, S193-228.

Lee MC, Orenstein MR, Richardson MJ, 2008. Systematic review of active commuting to school and children's physical activity and weight. J Phys Act Health 5, 930-949.

Nader PR, Bradley RH, Houts RM, McRitchie SL, O’Brien M, 2008. Moderate-to-vigorous physical activity from ages 9 to 15 years. JAMA 300, 295-305.

Ogden CL, Carroll MD, Curtin LR, Lamb MM, Flegal KM, 2010. Prevalence of high body mass index in US children and adolescents, 2007-2008. JAMA 303, 242-249.

Oreskovic NM, Winickoff JP, Kuhlthau KA, Romm D, Perrin JM, 2009. Obesity and the built environment among Massachusetts children. Clin Pediatr 48, 904-912.

Papas MA, Alberg AJ, Ewing R, Helzlsouer KJ, Gary TL, Klassen AC, 2007. The built environment and obesity. Epidemiol Rev 29, 129-143.

Perrin JM, Bloom SR, Gortmaker SL, 2007. The increase of childhood chronic conditions in the United States. JAMA 297, 2755-2759.

Quigg R, Gray A, Reeder AI, Holt A, Waters DL, 2010. Using accelerometers and GPS units to identify the proportion of daily physical activity located in parks with playgrounds in New Zealand children. Prev Med 50, 235-240.

Troiano RP, Berrigan D, Dodd KW, Masse LC, Tilert T, McDowell M, 2008. Physical activity in the United States measured by accelerometer. Med Sci Sports Exerc 40, 181-188. 